\title{
ADAPTACIÓN DE LA ESCALA HEDÓNICA FACIAL PARA MEDIR PREFERENCIAS ALIMENTARIAS DE ALUMNOS DE PRE-ESCOLAR
}

\section{FACIAL HEDONIC SCALE ADAPTATION TO MEASURE FOOD PREFERENCES AMONG PRESCHOOL CHILDREN}

\author{
Semíramis Martins Álvares $\mathrm{D}^{1 *}$, Julián Zapico $\mathrm{T}^{2}$, José Augusto de Aguiar Carrazedo $\mathrm{T}^{3}$ \\ ${ }^{1}$ Faculdade de Nutrição, Centro de Ciências da Vida, PUC-Campinas. Brasil. \\ ${ }^{2}$ Facultad de Veterinaria, Universidad de León. Brasil \\ ${ }^{3}$ Disciplina de Nutrologia, EPM/UNIFESP. Brasil.
}

Este trabajo fue recibido el 22 de Octubre de 2007 y aceptado para ser publicado el 14 de Enero de 2008.

Dirigir la correspond encia a:

Profesora

Semíramis Martins Álvares Domene

Faculdade de Nutrição, CCV

Pontifícia Universidade Católica de Campinas

Av. John Boyd Dunlop s/n 13059-900

Campinas, SP, Brasil.

semiramis@puc-campinas.edu.br

*Institución a que se debe atribuir el trabajo: Faculdade de Nutrição, Centro de Ciências da Vida, PUC -

Campinas. 


\begin{abstract}
The assessment of food acceptance during childhood is useful to provide educational guidelines. Scanty research has been done to validate hedonic facial scales for testing pre-schooll age children. Sensory tests were conducted in 2924 to 6 year old pre schoolage children with high (A) and low family income from a poor areas (B), using good tasting food (b) and food with modified flavor $(d)$. Food preference was assessed to validate the three point hedonic facial scale designed to identify gender differences. By weighting the food rejected, a $10 \%$ amount was considered as a limit for food acceptance. Results showed that there is a correlation between food acceptance and level of satisfaction (Ab, $p=0,033 ; A d, p<0,001)$, but no correlation was detected with children from poor areas $(B b, p=0,325$ and $B d, p=0,086)$. The hedonic facial scale must be improved in order to effectively assess pre schooll preferences.
\end{abstract}

Key words: sensory evaluation, pre-schooll, food preferences, food habits, income.

\title{
1. INTRODUCCIÓN
}

Las reacciones que ocurren en el consumo de alimentos están provocadas por el grado de satisfacción del consumidor con respecto a estímulos de los sentidos: olfato, sabor, tacto, vista y hasta la audición (1). El análisis sensorial es una disciplina que se dedica a estudiar formas de sistematizar estas observaciones, teniendo en cuenta la subjetividad que determina cuando un alimento es o no aceptado. Considerando que la opción por un alimento está muy influenciada por el placer que provoca, sobre todo en los niños (2), el desarrollo de pruebas de análisis sensorial, adaptadas al perfil de cada consumidor, tiene importancia para identificar preferencias o rechazos relacionados a la creación de hábitos y prácticas alimentarias y también, para la 
optimización de los recursos destinados a la adquisición de alimentos para las políticas públicas en el área de la alimentación y nutrición.

En la población infantil, la preferencia por uno u otro alimento está determinada por un conjunto complejo de estímulos sensoriales y culturales y no sólo por la predilección por sabores simples, como dulce o salado; este hecho revela que la estructura de las opciones alimentarias depende de bases biológicas; pero también educacionales (3). En este sentido, el papel de los educadores de la familia es decisivo para ampliar la gama de alimentos bien aceptados por los niños, que formulan sus preferencias sensoriales a partir de los 2 a 3 años de edad (4).

La medición de la aceptación del alimento por pre-escolares es complicada debido a la falta de instrumentos de análisis sensorial adaptados a este grupo de población, que presenta una estructura de percepción sensorial diferente de los adultos (5).

La obtención de respuestas confiables en pruebas sensoriales depende del grado de madurez del niño para interpretar adecuadamente las instrucciones recibidas, lo que parece ser mejor a partir de los 5 años, por comparación con resultados obtenidos con niños de 3 años (6). La prueba de escala hedónica es una de las utilizadas con adultos. A partir de ella se ha desarrollado una prueba de escala hedónica facial para uso con niños y adultos no alfabetizados $(7,8)$. Sin embargo, la fiabilidad de la información obtenida es cuestionable, toda vez que la simpatía por la figura, y no la expresión de satisfacción por el consumo de alimento, sería el agente determinante de la elección del niño (9). De hecho, el uso de la escala hedónica facial de siete puntos (10), para evaluar la aceptación de alimentos agradables al paladar, en un estudio realizado con pre-escolares, demostró que las figuras "alegres" eran las preferidas, independientemente del grado de satisfacción experimentado por el degustador (11). 
El presente estudio tiene como objetivo evaluar, por medio de un estudio de validación, la asociación entre la aceptación del alimento por pre-escolares y el registro del grado de satisfacción de los mismos empleando la escala hedónica facial de tres puntos.

\section{SUJETOS Y MÉTODO}

\subsection{Muestra}

El estudio se llevó a cabo mediante el modelo de escala hedónica facial propuesta por Mori en 1993 (12), adaptada para tres puntos para su uso con pre-escolares de 4 a 6 años matriculados en escuelas del municipio de Campinas (SP, Brasil), cuya participación fue debidamente autorizada por sus padres o responsables. Se obtuvo la aprobación del Comité de Ética en Salud de la Universidad. Para eliminar la eventual interferencia del poder adquisitivo sobre el juicio y/o aceptación, las pruebas se realizaron en escuelas públicas de zonas pobres de la periferia y escuelas privadas situadas en regione s centrales del municipio.

Se realizaron 292 pruebas de análisis sensorial con alumnos de la misma franja de edad, e de dos estratos socioeconómicos: 131 con niños de alto poder adquisitivo (grupo A), 64 con alimento de buen sabor $(\mathrm{Ab})$ y 67 con alimento de sabor diferenciado, con el fin de provocar insatisfacción (Ad); otras 161 pruebas fueran realizadas con niños de bajo poder adquisitivo (grupo B), 81 con alimento de buen sabor (Bb) y 80 con alimento de sabor diferenciado (Bd)

\subsection{Estudio de escala y medida de aceptación de los alimentos}

La validación de la escala consistió en la medición de la aceptación del alimento mediante el peso de la sobra sucia, o el resto. Se consideró que hubo buena aceptación del alimento todo consumo superior al $90 \%$ ofrecido (13). 
Los escolares recibieron alimento de buena aceptación (b), con objeto de provocar satisfacción, o alimento de mala aceptación (d), formulado para provocar insatisfacción. El alimento (b) fue una gelatina de frutas; el (d) la misma gelatina con sólo un 10\% de contenido de azúcar respecto a la primera.

La escala evaluada consta de tres puntos con caracterización de género (Anexo 1). Se asignó cada punto con la siguiente calificación: el primer punto de esta escala corresponde a la opción, "gustó", el segundo a la "no gustó y no disgustó" y el tercero, "no gustó". Las pruebas fueron realizadas en los comedores escolares en horario reservado exclusivamente para esta actividad, y la explicación acerca del significado fue dada por el mismo profesional. La verificación de algún tipo de asociación entre la aceptación del alimento y el registro del grado de satisfacción del pre-escolar degustador fue evaluado por medio del test del Qui-cuadrado - ?2 $(a=0,05)$.

\section{RESULTADOS Y DISCUSIÓN}

La evaluación de las preferencias alimentarias en la infancia por medio de escalas desarrolladas para adultos, puede generar distorsión en los resultados obtenidos, al no considerar la diferencia entre los sistemas bioquímicos de la percepción del sabor entre los dos gr upos (5), o por emplear instrumentos inadecuados a la madurez necesaria para comprender las instrucciones y la forma exigida por las pruebas para que el degustador exprese correctamente su grado de satisfacción (14), o el efecto de la afectividad sobre el juicio y la aceptación (15).

Los resultados obtenidos en el presente trabajo, analizados estadísticamente por el procedimiento de ?2, se muestran el las tablas 1 y 2 . Todos los niños responderán a la prueba sin dificultad.

La primera corresponde a la muestra regular (b), alimento de buena aceptación. 
TABLA 1

Resultados de la prueba de validación de la escala hedónica facial de tres puntos en pre escolares de dos estratos socioeconómicos (A y B) y alimento de buena aceptación (b).

\begin{tabular}{|c|c|c|c|c|c|c|c|c|}
\hline & \multicolumn{4}{|c|}{ Bajo poder adquisitivo $(\mathrm{Bb}, \mathrm{n}=81, \mathrm{p}=0.325)$} & \multicolumn{4}{|c|}{ Buen poder adquisitivo $(\mathrm{Ab}, \mathrm{n}=64, \mathrm{p}=0.033)$} \\
\hline & \multicolumn{2}{|c|}{ Aceptación > 10\% } & \multicolumn{2}{|c|}{ Aceptación $\leq 10 \%$} & \multicolumn{2}{|c|}{ Aceptación > 10\% } & \multicolumn{2}{|c|}{ Aceptación $\leq 10 \%$} \\
\hline & $\mathrm{n}$ & $\%$ & $\mathrm{n}$ & $\%$ & $\mathrm{n}$ & $\%$ & $\mathrm{n}$ & $\%$ \\
\hline Gustó & 64 & 79 & 5 & 6 & 38 & 59,5 & 16 & 25 \\
\hline No gustó/ no disgutó & 7 & 8,5 & 2 & 2,5 & 3 & 4,5 & 1 & 1,5 \\
\hline No gustó & 3 & 4 & 0 & 0 & 1 & 1,5 & 5 & 8 \\
\hline Total & 74 & 91,5 & 7 & 8,5 & 42 & 65,5 & 22 & 34,5 \\
\hline
\end{tabular}

La segunda corresponde a la muestra modificada con sabor diferenciado (d), formulada con el objetivo de provocar insatisfacción. 
TABLA 2

\section{Resultados de la prueba de validación de la escala hedónica facial de tres puntos con pre-escolares de dos estratos socioeconómicos (A y B) y alimento de sabor diferenciado (d).}

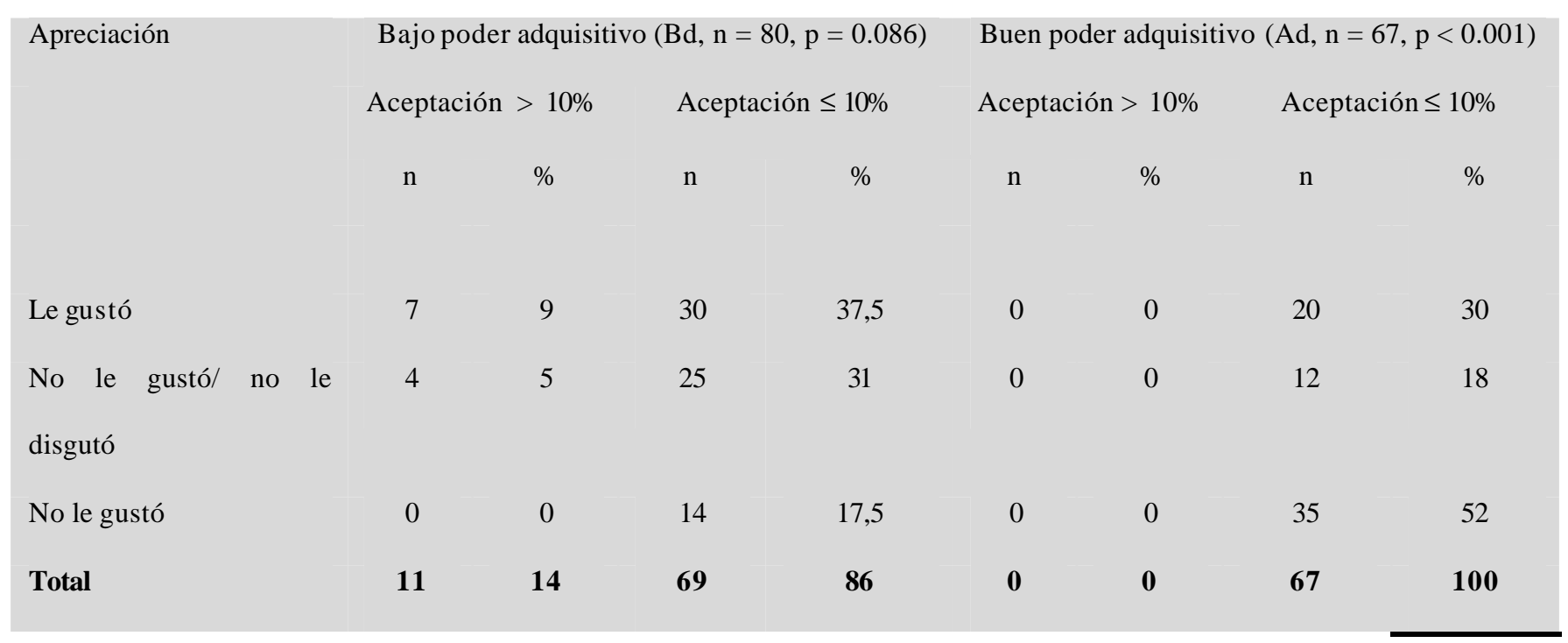

La formación de la estructura de las preferencias alimentarias durante la infancia está fuertemente influenciada por la oportunidad de acceso a los alimentos; así la gama de opciones experimentadas en esta época puede condicionar el repertorio de preferencias en la adolescencia y en la vida adulta. La importancia de la influencia de las madres sobre la alimentación de los hijos fue analizada por Skinner y cols. (16) en un estudio longitudinal con 70 niños y sus padres; los autores demonstraran no existir alteración del número de alimentos bien aceptados entre 4 y 8 años de edad y que había limitación en el número de ellos dependiendo de la influencia de las madres. Estos resultados son semejantes a los encontrados en Brasil por Maciel y col. (17), que demostraron la influencia de las madres sobre sus hijos por la preferencia de sabores dulces.

En relación a la salud, un menor número de alimentos aceptados contribuye a una dieta poco variada en nutrientes. Según el trabajo de Calfas y cols (18), la influencia de los padres 
parece ser menos importante sobre la adopción de buenos hábitos de salud y a la vez, que no hay consenso sobre el tema.

La utilización de la escala de tres puntos, con diferenciación de género, demostró una mejor correlación que la escala de siete puntos utilizada en otro estudio (11), realizado en las mismas condiciones de aceptación considerada por medio del peso de las sobras; pero sólo hubo diferencias estadísticamente significativas con pre-escolares de buen poder adquisitivo (tabla 1, p $=0.033$ para $\mathrm{Ab}, \mathrm{y}$, tabla $2, \mathrm{p}<0.001$ para Ad, respectivamente). Además, es importante una cuidadosa y correcta aplicación de la escala, tal como realizarla en ambiente aislado para pequeños grupos, preferentemente sin la presencia del profesor, aunque su presencia puede ser recomendable para transmitir confianza y tranquilidad en situaciones de desconfianza de los niños hacia el entrevistador. El modelo, entretanto, no fue adecuado para su aplicación a población de bajo poder adquisitivo (tabla 2, $\mathrm{p}=0.325$ para $\mathrm{Bb}$ y 0.086 para $\mathrm{Bd}$, respectivamente).

La fuerte asociación entre preferencias de alimentos y consumo, sugiere que la introducción precoz de alimentos variados en la dieta, puede aumentar la gama de alimentos bien aceptados en la infancia (19). En revisión sobre los determinantes psicológicos propuestos para explicar patrones de consumo de alimentos, Eertmans cols. (20) realizan una crítica a los modelos de educación alimentaria basados en la información sobre riesgo y beneficio que no consideran el papel del aprendizaje continuado. A pesar de los pocos estudios sobre el tema, el uso de escalas adaptadas a la población pre-escolar, que también puede ser empleada con adultos no alfabetizados, es una forma adecuada como complemento de formación de la estructura de preferencias de alimentos en la infancia, convirtiéndose así, en un instrumento útil para la dirección de intervenciones educativas precoces, con impacto efectivo sobre los hábitos futuros en materia de alimentación, por actuar en la fase de desarrollo. 
Alimentos en principio rechazados por los niños, si son identificados y ofrecidos repetidamente, pueden pasar a ser deseados y consumidos. Wardle y cols (21), estudiando durante dos semanas un grupo de niños, observaron las preferencias y el consumo de alimento, previamente rechazado, significativamente mayores en el grupo al que fue ofrecido por 8 veces, que en el grupo control. Adecuadamente identificados, por medio de pruebas sensoriales, alimentos nutritivos no aceptados pueden ser priorizados para una propuesta educativa a largo plazo, contribuyendo efectivamente a la incorporación de prácticas alimenticias saludables, con beneficios inequívocos para la salud de las personas en su adolescencia y vida adulta.

\section{CONCLUSIÓN}

La aplicación de la escala hedónica facial de tres puntos, con identificación de género, puede ser un instrumento útil para ayudar a la identificación de las preferencias y rechazos de alimentos en la etapa pre-escolar en grupos de buen poder adquisitivo, pero no es adecuada para aplicación en grupos de bajo poder adquisitivo.

\section{RESUMEN}

El conocimiento de la aceptación de los alimentos en la infancia es un recurso útil para orientar intervenciones educativas. No existen estudios de validación de escala hedónica facial para medida de aceptación con niños. Se realizaron 292 pruebas con pre-escolares de 4 - 6 años de buen poder adquisitivo (A) o residentes en bolsones de pobreza (B), con alimento de buena aceptación (b) y de sabor modificado (d). La medida de aceptación del alimento consideró hasta el 10\% del peso ofrecido. Los datos fueran evaluados por medio de test del Qui-cuadrado. Hubo correlación entre la aceptación del alimento y el registro del grado de satisfacción del degustador ( $\mathrm{p}=$ 0,033 para $\mathrm{Ab}, \mathrm{y} \mathrm{p}<0,001$ para $\mathrm{Ad}$ ), pero no para el grupo residente en bolsones de pobreza 
$(\mathrm{p}=0,325$ para $\mathrm{Bb}$ y $\mathrm{p}=0,086$ para $\mathrm{Bd})$. La escala hedónica facial de tres puntos debe ser mejorada para su uso con pre-escolares.

Palabras claves: análisis sensorial, pre-escolares, preferencias alimenticias, hábito de alimentación, poder adquisitivo.

Agradecimientos: Los autores agradecen a FAPESP la financiación del proyecto del que forma parte este trabajo (Proc.98/14020-1). A Bianca Massucheli Chimenti y Viviane Fusco, por su colaboración en la recolección de los datos, así como a los docentes, y a los pre escolares incluidos en el grupo estudiado.

\section{BIBLIOGRAFIA}

1 Koivisto H. Factors influencing children's food choice. Ann Med 1999; 31:26-32.

2 Hursti UK. Changing food habits in children and adolescents. Experiences from intervention studies. Au Scand Jr Nutr 1998; 42:39-41.

3 Wardle J, Herrera ML, Cooke L, Gibson EL. Modifying children's food preferences: the effects of exposure and reward on acceptance of an unfamiliar vegetable. Eur J Clin Nutr 2001; 57:341 8.

4 Nicklas TA, Baranowski T, Baranowski JC, Cullen K, Rittenberry L, Olvera N. Family and child-care provider influences on preschool children's fruit, juice, and vegetable consumption. Nutr Rev 2001;59:224-35.

5 Liem DG, Mennella JA. Heightened sour preferences during childhood. Chem Senses 2003; 28:173-80. 
6 Guthrie CA, Rapoport L, Wardle J. Young children's food preferences: a comparison of three modalities of food stimuli. Appetite 2000; 35:73-7.

7 Bergara Almeida S, Da Silva M A A P. Hedonic scale with reference: performance in obtaining predictive models. Food Qual. Pref 2002; 13:57-64.

8 Sparkman AF, Simmons LF, Sullivan S, Roberts-Gray C. Tools to measure sensory appeal of menus planned for children. J Am Diet Assn 1998; 88:488-491.

9 Stone H, Sidel JL. Sensory evaluation practices. Academic Press, San Diego, 1985.

10 Kimmel SA, Sigman-Grant M, Guinard J. Sensory testing with young children. Food Tech 1994; 4:92-99.

11 Domene SMA, Veiga FM, Marino C, Assumpção AM, Zabotto CB, Vítolo MR. Validação de metodologia para análise sensorial com pré-ecolares. Rev Ci Med 2002; 11:131-8.

12 Almeida TC, Hough G, Damásio MH, da Silva MAAP. Avanços em análise sensorial. São Paulo, Ed. Varela, 1999.

13 Lawlees HT. Getting results you can trust from sensory evaluation. Cer Foods World 1994; 39:809-14.

14 Wardle J, Sanderson S, Leigh Gibson E, Rapoport L Factor-analytic structure of food preferences in four-year-old children in the UK. Appetite 2001;37:217-23.

15. Kim J, Morris JD. The power of affective response and cognitive structure in product-trial attitude formation. J Advertising 2007; 36: 95-106,.

16 Skinner JD, Carruth BR, Wendy B, Ziegler PJ. Children's food preferences: a longitudinal analysis. J Am Diet Assoc 2002; 102:1638-472.

17 Maciel SM, Marcenes W, Watt RG, Sheiham A The relationship between sweetness preference and dental caries in mother/child pairs from Maringa-Pr, Brazil. Int Dent J 2001; $51: 83-8$ 
18 Calfas KJ, Sallis JF, Nader PR. The development of scales to measure knowledge and preference for diet and physical activity behavior in 4- to 8-year-old children. J Dev Behav Pediatr 1991;12:185-90.

19. Pérez-Rodrigo C, Ribas L, Serra-Majem L, Arance J. Food preferences of Spanish children and young people: the enKid study. Eur J Clin Nutr 2003; 57:S45-S48.

20 Eertmans A, Baeyens F, Van Den Bergh O. Food likes and their relative importance in human eating behavior: review and preliminary suggestions for health promotion. Health Educ Res $2001 ; 16: 443-56$.

21 Wardle J, Sanderson S, Leigh Gibson E, Rapoport L. Factor-analytic structure of food preferences in four-year-old children in the UK. Appetite 2001;37:217-23. 
Modelo de escala hedónica facial (modificación de la propuesta por Mori, 1993).

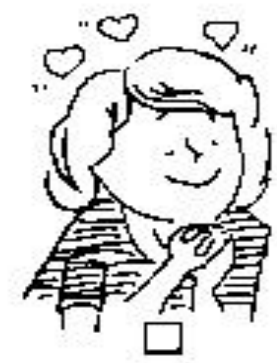

Opción 1

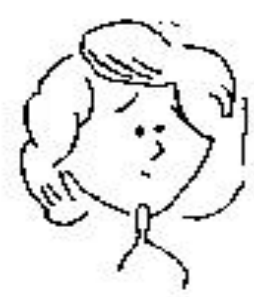

$\square$

Opción 2
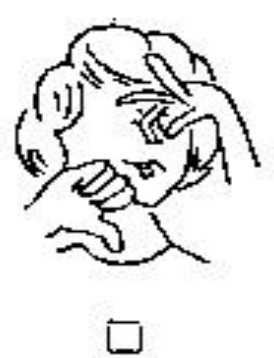

Opción 3

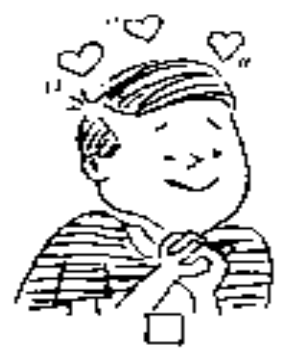

Opción 1

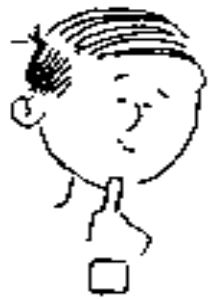

Opción 2

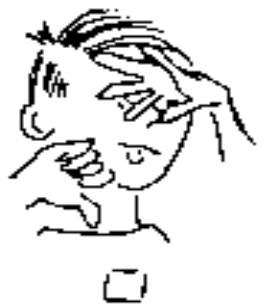

Opción 3 